\title{
ULTRA HIGH ENERGY COSMIC RAY SOURCES \& EXPERIMENTAL RESULTS
}

\author{
Peter L. Biermann ${ }^{1,2}$, Gustavo Medina Tanco ${ }^{3}$ \\ ${ }^{1}$ Max-Planck Institute for Radioastronomy, Bonn, Germany \\ ${ }^{2}$ Department for Physics and Astronomy, \\ University of Bonn, Germany \\ ${ }^{3}$ Instituto Astronomico e Geofisico \\ Universidade de Sao Paulo, Brasil \\ plbiermann@mpifr-bonn.mpg.de,gustavo@astro.iag.usp.br
}

\begin{abstract}
Here we discuss the latest developments in the debate, where the ultrahigh energy cosmic ray particles come from. In this brief review, we emphasize the predictions that necessarily follow from the various concepts proposed. We discuss both sources and propagation, and spend some space on the by now most conservative model, giant radio galaxies and their hot spots and jets, because it allows more steps in the reasoning to be checked, and is more tightly constrained than any other model. It has survived about forty years of debate already. We summarize the task ahead of us at the end, including the development of new technologies to observe ultra high energy cosmic rays, e.g., with the radio emission of air showers, which may lead to an alternative to the fluorescence detectors with their small duty cycle. Due to HiRes, AUGER, and the plans for EUSO, the future is bright, and the ultra high energy cosmic ray particles may yet allow us to explore new physics.
\end{abstract}

\section{Introduction}

Over the last few years controversies have arisen both about the experimental results, and also about the theoretical interpretations. We are going to review the situation, but will do so in very limited space.

\subsection{The cosmic ray spectrum}

The cosmic ray spectrum in nuclei shows the following basic properties:

- approx. $E^{-2.7}$ until the knee

- a bend downwards at around $310^{15} \mathrm{eV}$

- approx. $E^{-3.1}$ beyond the knee, with a slight downward dip from $310^{17} \mathrm{eV}$

- a transition near $310^{18} \mathrm{eV}$, and then approx. $E^{-2.7}$ again
- uncertainty beyond $510^{19} \mathrm{eV}$, either mild cutoff (HIRES) or a continuation (AGASA)

A main reference for a collection of data until 1997 is in [129. General references are in 5352 1427. Classical reviews are, e.g., in [71, while some recent reviews are 199420873065 .

Generally we emphasize very recent work, and suggest the reader consult recent reviews for older references.

\subsection{Interactions in the microwave back- ground}

The ultra high energy cosmic ray particles interact with the microwave background, and their losses lead to an ubiquitous cutoff, usually referred to as Greisen-Zatsepin-Kuzmin cutoff, after its discoverers, under some simplifying assumption about the nature of these particles (pro- 
tons), the nature of intergalactic propagation, as well as source distribution.

- pair and pion production

- limits effective distance from which a high energy proton (or neutron, or nucleus) may come

- discovered by K. Greisen, G. Zatsepin and V. Kuzmin

- GZK distance about $50 \mathrm{Mpc}$, a travel distance

- what sources?

The key references are 6313194. A recent generalization to nuclei is in 45464716 . A popular account is in 40].

\section{Galactic Cosmic Rays}

\subsection{Transition from Galactic Cosmic Rays?}

The newest data suggest and confirm that the transition from Galactic cosmic rays to an extragalactic population appears to be near $310^{18} \mathrm{eV}$.

- Near $310^{18} \mathrm{eV}$ transition

- below heavy to medium nuclei, above light nuclei

- spectral dip there seen by Fly's Eye

- now confirmed by AGASA, HiRes, Yakutsk,...

\subsection{Origin of Galactic Cosmic Rays}

A quantitative theory for Galactic cosmic rays was proposed in 1993, 232425120262928, with an earlier step [126]. Quantitative tests are now again possible with the KASKADE data, as well as the latest HiRes, Akeno, Yakutsk, and Haverah Park data. Detailed simulations have been carried out at several levels of sophistication by A. Vasile and S. Ter-Antonyan, and they show consistently, that the older proposals do give a suitable interpretation of the new data at all energies for Galactic cosmic rays. At the level of today's knowledge the older proposal thus does find confirmation in the new data. The suggestion as how to explain the knee, 99100, based on a slightly diminished efficiency of curvature drift in the supernova shock racing through a magnetic wind of a massive star, strongly points to a specific mechanism for the supernova, the mechanism proposed by G. Bisnovatyi-Kogan in 1970, based on earlier ideas by N. Kardashev from 1964, 8131. In these ideas the core of the star collapses to a small disk, held by the angular momentum barrier, and then magnetic fields transport energy and angular momentum to the envelope, exploding the star, and allowing final collapse of the core. Such a mechanism naturally gives the correct energies $-10^{52}$ erg is required for the explosion, and seen in Supernova 1998bw, 73 95]. If accepted, then this in turn suggests that all very massive stars come to a common final end at the point of supernova explosion, and there seems a possibility that they could become a new and very bright standard candle in cosmology - provided we find a correction for the extreme asphericity expected from the Bisnovatyi-Kogan mechanism. In this context it is also possible perhaps to understand the gamma ray emission of the Galaxy, 75] as well as the $\mathrm{B} / \mathrm{C}$ ratio energy dependence, 10528; as the general activity in the Galaxy in almost everything connected to young and massive stars is centered in the Galactic center region, one might expect that that region may serve as a paradigm for bot stellar and perhaps even non-stellar types of activity, 9192. Other concepts are explored in 363738 .

- consistent with (KASKADE, HiRes, Akeno,...):

- Red Supergiant (RSG) stars and Wolf Rayet (WR) stars explode, and Kolmogorov spectrum in Inter-Stellar Medium (ISM)

- knee from transition in efficiency of curvature drift acceleration

- final cutoff due to magnetic field in wind, Larmor limit

- consequences for Supernova mechanism, and possibly bright standard candle 
Details will be published in further papers.

\section{Homogeneous source distribution?}

The GZK cutoff is expected under several assumptions, one of which is a homogeneous source distribution. There are no astronomical sources known, which are truly distributed in a homogeneous fashion.

- The very high energies, above $310^{18} \mathrm{eV}$ :

- assume zero effect of magnetic field

- assume protons (or nuclei)

- assume an initial energy far beyond anything observed

- obtain a strong downturn feature: The GZK-cutoff expected near $510^{19} \mathrm{eV}$

The latest data from HiRes and AGASA do not confirm each other on whether the expected cutoff has been seen at all, and also do not clearly show whether the data are compatible with the simple cutoff, 7212210]. The very existence of events beyond $10^{20} \mathrm{eV}$ suggests that the very simple model cannot work, and one of the key points was the assumed homogeneous source distribution. Already a source distribution more closely consistent with bright and early Hubble type galaxies suggests a clearly higher expected flux near $10^{20} \mathrm{eV}$, e.g., 32.

\section{Sky distribution?}

For many years a controversy has raged on whether specific events do correlate with known sources; one of the earliest suggestions by Ginzburg was that the nearest powerful radio galaxy, M87, could be a good candidate - it still is. However, a direct positional coincidence of an event with a candidate source, or better, with an entire class of sources, has been argued repeatedly, 49. Then the claim by AGASA, that there are double and triple events, does allow, again under very simplifying assumptions, to obtain statistics on possible sources. Recent investigations of such correlations are 5954555758 and 4412312412562 .
- Assume no magnetic field

- then sky distribution: many sources

- detection of doubles and triplets: real sources

- are there sources there?

- with enough power? $L_{C R}=L_{\text {el.magn }}$ at most

- Using the deep radio surveys, there is always such a source

Under the proposal, that radio galaxies such as M87, a source, for which the observed optical spectrum suggests the presence of protons of $10^{21} \mathrm{eV} 22$, required to explain the very common cutoff in the optical synchrotron emission near $310^{14} \mathrm{~Hz}$, are good candidate sources, all radio galaxies, and a fortiori, all BL Lac type sources are candidates, see, e.g., [103. And, under the standard unification picture of active galactic nuclei, in the same vein, all flat spectrum radio sources are good candidates, under this by now most conservative model to explain ultra high energy cosmic rays. It is not surprising that such claims have been tested, in various papers such as 49], and now, in a new series of papers by $P$. Tinyakov \& I. Tkachev, 4412312412562 , and others, [117. All these papers find tantalizing hints of a possible correlation between sources in this class, and observed cosmic ray events; however, the interpretation requires uncharged particles with a penetrating power through the bath of the microwave background far in excess of what protons can do. Such particles may well exist, but there is no self-consistent theory for them at this time. Also the statistics of source correlations have not been confirmed to everybody's satisfaction. On the other hand, any highly significant correlation of even a subset of all observed events with a known class of sources is interesting, and a clear clue to any real source, as well as to the nature of particle.

The Yakutsk data and an extensive discussion of the array and an interpretation of its data have been published in a series of papers, 615960545556575893 , but the conclusion is 
not ultimately clear; we need to understand the experiment better in order to compare its important results with those of other experiments.

\section{Magnetic Fields}

Magnetic fields in the universe are known to exist, now for over fifty years, 8284, and they seem to be just about everywhere, 110 83 39.

- Magnetic fields have been detected

- they are strong

- they are highly inhomogeneous

- they compete with other forms of energy

- only in cosmic clusters, filaments and sheets,

- but not in the voids, and not overall

- 5-10 microGauss in clusters - at least

- 0.01 to 1 microGauss in filaments and sheets

- bending of paths of energetic particles

Therefore, magnetic fields cannot be ignored in the propagation of energetic particles, and even at the highest energies observed the cosmic magnetic fields have a noticeable effect. In fact, magnetic fields correlate well with the cosmological galaxy distribution, and so are relatively strong in the sheets and filaments of the galaxy distribution, and so also in the sheet connecting us to the Virgo cluster. For any particles emitted by some source in the general region of the Virgo cluster - such as M87 - the arrival directions outside the Galaxy are expected to cluster along the supergalactic plane then, and so represent an elongated band in the sky - before they pass into the magnetic field surrounding our Galaxy. Recent work is in 66676811369707613016 .

\subsection{Galactic Magnetic Field}

Right after the discovery of the Solar wind in 1950, 21], the suggestion came up that our Galaxy may also have a wind. If our Galaxy has a wind, it is likely that it is driven by cosmic rays, because they need to deposit their energy lost in adiabatic losses into the surrounding magnetic medium, and that easily drives a wind, e.g., 33 34 104 35]. The driving of a magnetic wind by cosmic rays has some similarity to the possible influence of radiation driving a magnetic wind, 111. Such a wind implies that the magnetic field topology right from the start just above the hot thick disk is in the asymptotic E. Parker from of 1958, 98, because otherwise the wind would take so much angular momentum out from the gas in the disk, that the Galaxy would long have stopped all its star formation - all the gas would have been deposited in the central region, and to some small measure in the central black hole. This implies the topology of the magnetic field in the wind:

- If Galaxy has wind

- driven by cosmic rays, and supernova shocks

- then magnetic field topology as in Solar Wind

- Archimedean Spiral, i.e. $B_{\phi} \sim \sin \theta / r$

- turbulence spectrum $k^{-2}$ in wavenumber for adopted isotropy in wave number phase space, derived from the saw-tooth pattern of shock driving

- if so, then power of wind should scale with 60 micron luminosity, since Cosmic Ray activity scales with star formation, and star formation is visible at 60 micron

It can be shown, that all such winds can easily provide the universe with all the observed magnetic fields, 110834. If we could only understand where galaxies really get their magnetic fields from, we would be much better shape. Magnetic fields will be discussed elsewhere.

\section{Predictions: Double and triple events}

In this paper, we wish to emphasize the predictions, that arise from the various source and propagation arguments.

In such a picture of a magnetic wind of the Galaxy, there is one obvious explanation for the 
doubles and triples observed by AGASA: magnetic lensing, or in other words, the caustics given by the propagation of charged particles through a magnetic wind of the Galaxy; these caustics surely overlap for different particle energies. Doubles and triples can occur when there are two possible paths for particles at very different energies, leading to the same direction observed at Earth, but coming from different sources in the sky, presumably a band in the sky in arrival direction as seen outside the Galaxy. For this argument it does not actually matter, where exactly the particles are coming from. Recent work on this is in 6869121 .

- Magnetic caustics: $E_{2} / E_{1}$ either near 1 or from about 2-3

- Then galactic wind required, driven by cosmic rays

- and permeated by a relatively strong magnetic field

- Sources: Reasonably sampled continuous spectrum - implies neutral particles

- Requires strong source

\section{Radio galaxies, Microquasars, Gamma Ray Bursts}

Diffusive shock acceleration is one commonly accepted now classical mechanism to accelerate charged particles, based on the original ideas of E. Fermi $(1949,1954) 50511385437814$. At a shock the magnetic irregularities on both sides of the shock play a relativistic tennis game with the charged particles, and, just as in real life occasionally drop a "ball", i.e. a particle, which then escapes downstream from the shock. In is most simple form this process produces an $E^{-2}$ spectrum.

- (weakly) relativistic shock wave acceleration

- $10^{21} \mathrm{eV}$ proton energy in the jet of M87 required by optical observations, 22 .

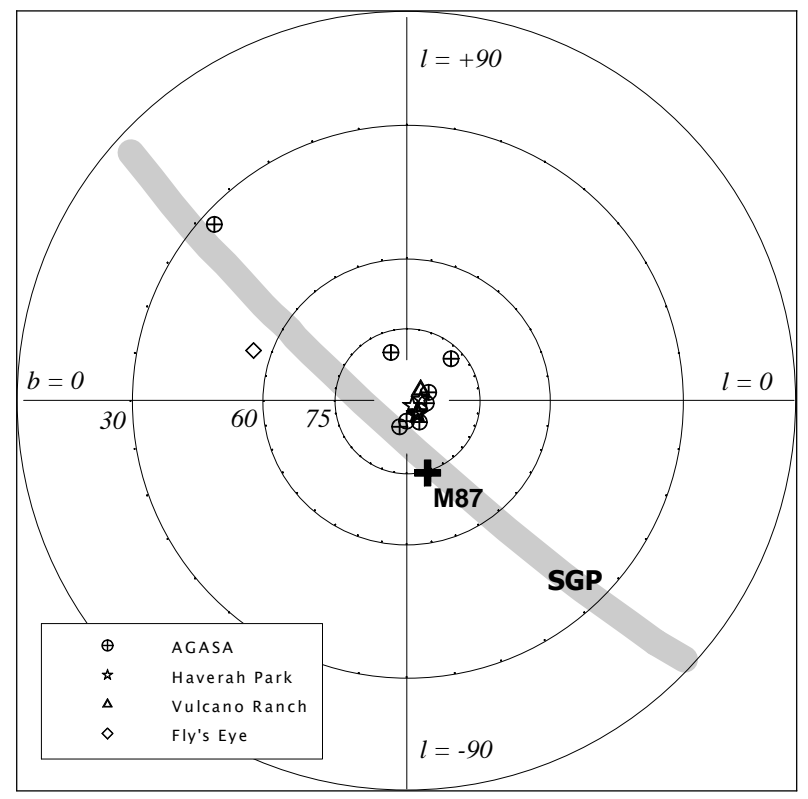

Figure 1. In this graph we show the arrival directions of observed cosmic rays calculated back to outside the assumed Galactic wind model with a Parker like magnetic field topology 


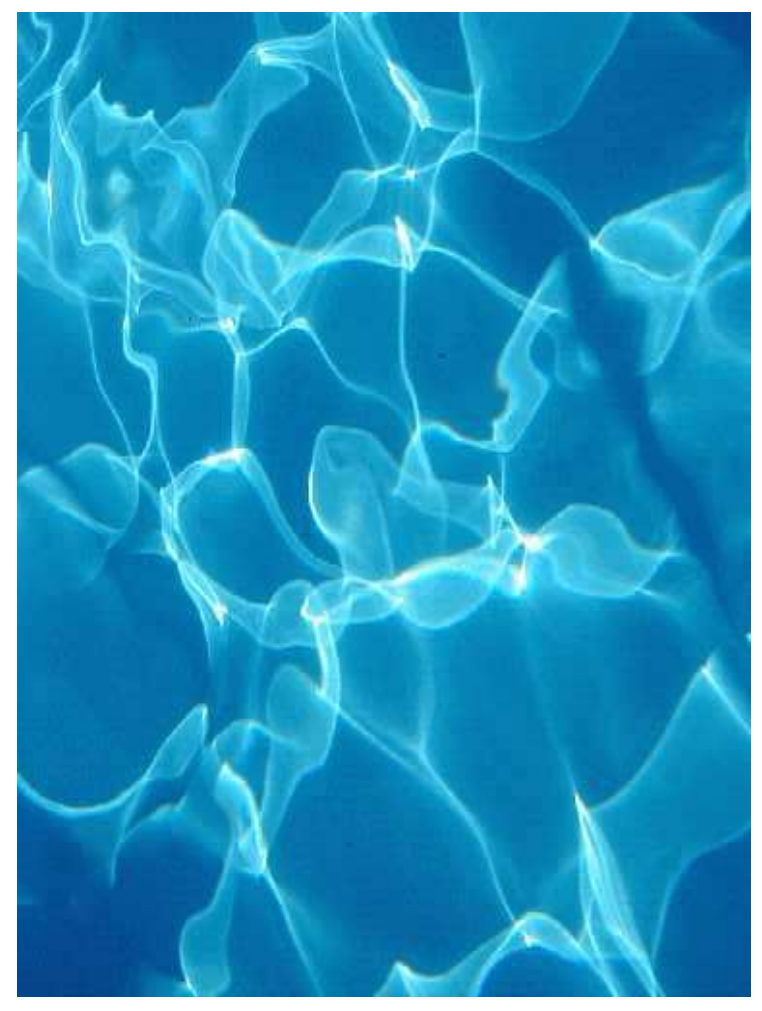

Figure 2. In this graph magnetic lensing is shown in a simulation based on sunlight shining on a swimming pool, and then considering the pattern of light at the bottom of the pool. This clearly shows the caustics in the imaging, and the peaks of light, corresponding possibly to the doubles and triples of events observed. Here we need to imagine a similar pattern, derived basically by scaling; then the superposition of such patterns at various scalings to represent different particle energies allows overlaps, which could be interpreted as those sites where we have mutliple events at very different particle energies. This graph is due to E. Roulet

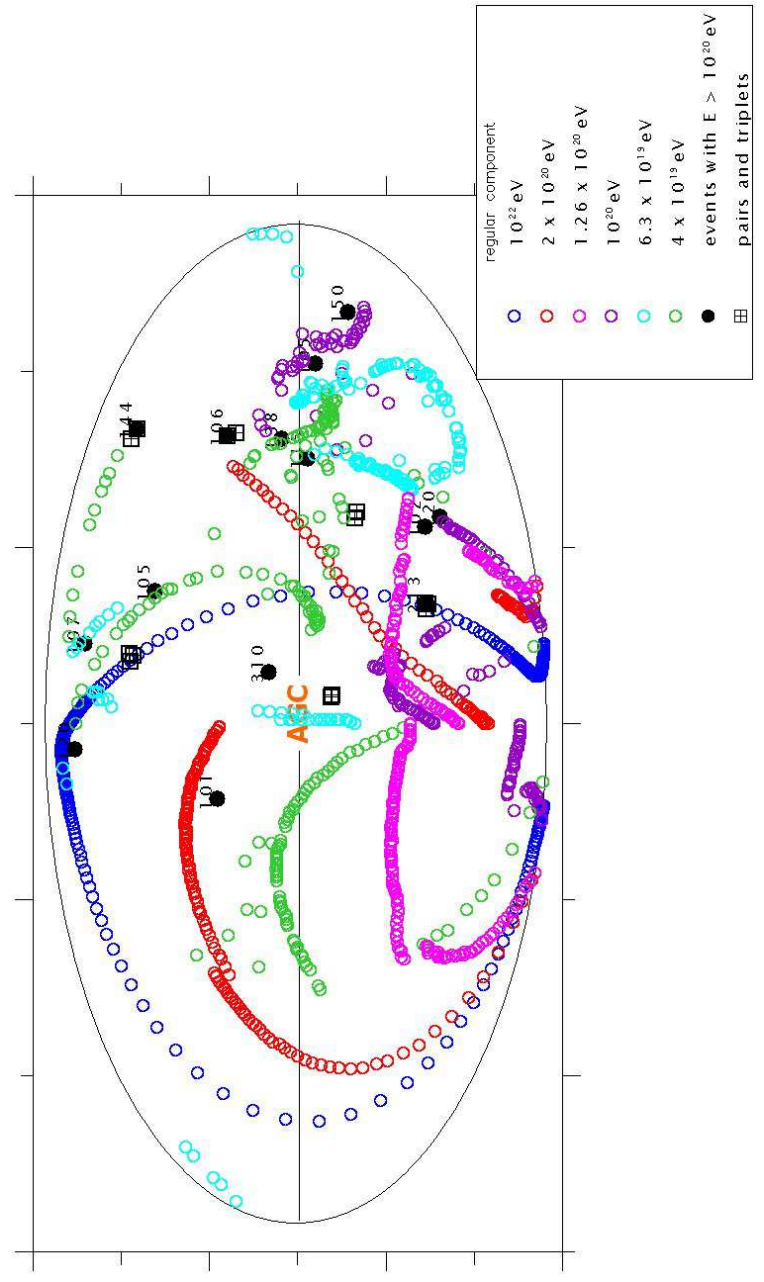

Figure 3. In this graph we show the arrival directions of cosmic rays at various energies, coming from the supergalactic plane in a long band, and then down to us through a Galactic wind modeled after Parker again. At the highest energy, very little bending is shown - a factor of 30 above the maximum energy observed. As then the energy of the particle used comes down, the bending becomes first noticeable, and then strong, leading to overlaps between different distorted bands. These overlaps then can directly be interpreted as locations on the sky, where multiple events with very different energies are likely to occur. In fact, the observed multiple events are already well approximated in this simple scheme. 
- as in many Active Galactic Nuclei (Jets, Hot Spots, or compact sources)

The maximum particle energy scales with the root of the power in the jet, 88, and so low power sources such as Microquasars cannot yield really high energies; this can be compensated by relativistic boosting, as in Gamma Ray Bursts, [101, but then the observed fluence distribution does not give a sufficient number of such sources in cosmological proximity to explain either the bulk of Galactic cosmic rays nor extragalactic cosmic rays, 106 107108. A different point of view is in 12742]. A variant on this topic is to consider magnetars, 8, or pulsars, 11. Such stellar sources may also contribute here, but with the particles coming from other galaxies; various options are discussed, e.g., by [119]. Radiogalaxies are considered again in a simple model in [15].

\subsection{Predictions: Microquasars and Gamma Ray Bursts}

Even though it appears unlikely hat microquasars and Gamma Ray Bursts do provide the bulk of the observed cosmic rays at any energy, they are clearly sources of energetic particles, and as such should contribute at some level; as they almost certainly have a different spectrum, and a different source distribution compared to, e.g., large early Hubble type galaxies, their flux contribution should be examined. It might be visible in some special feature in cosmic rays, not yet ascertained, in the gamma ray spectrum from galaxies, or in neutrinos - the best way to connect to specific sources. We have a fairly good handle on the physics of microquasars from the work by S. Markoff and G. Romero - we need to build on this understanding, e.g., 90109.

- also sources of energetic particles

- if pointed at us, very interesting

- unlikely to contribute at the highest energies

- doubtful to dominate at lowest energy

- could be checked in neutrinos

Recent work on the neutrino aspect is in 35, 41.

\section{Predictions: M87}

To be specific, the proposal has been made 22, based on optical spectra, that M87 not only requires protons in the source at $10^{21} \mathrm{eV}$, but also, that M87 may be the dominant source to explain the observed ultra high energy cosmic rays. Recent work on M87 is in 103. This leads to some clear predictions:

- M87: all events should point back to Supergalactic Plane Sheet - to within the noise produced in the propagation through the $k^{-2}$ turbulence spectrum in magnetic irregularities

- arrival directions seen outside our Galaxy elongated along sheet

- transition to light elements $(\mathrm{H}, \mathrm{He})$ near and above $310^{18} \mathrm{eV}$

- and such all the way to maximum energy

Clearly, M87 cannot be blamed for everything, but at the maximum energy there may only be one source remaining among all the contributors, and a suggestion is that this last remaining single source is M87. Weaker sources perhaps are other lower power radio galaxies such as Cen A, see [77, which are expected to contribute flux to about $510^{19} \mathrm{eV}$, from the relation between jet power, magnetic fields and maximum particle energy already quoted above. Of course, it has become obvious from the latest simulations that radio galaxies are highly time dependent in all their manifestations, and so we need to keep the option open, that such radio galaxies were more powerful at the time of particle production; their past behaviour can probably be checked with X-ray data.

\subsection{Predictions: Galactic wind}

The suggestion that M87 is the dominant source requires that our Galaxy has a powerful magnetic wind:

- implies Galactic wind with large size - 1/2 to $1 \mathrm{Mpc}$ 
- think of all galaxies with similar cosmic ray power, as measured through star formation rate, and such through their far infrared luminosity

- all similar galaxies should show such a wind bubble

- relatively strong magnetic field

- mass flow large, energy flow large, angular momentum flow small, with a wind shell (observable in Lyman alpha absorption)

- the most important test of such a picture is to avoid the bottle effect, thereby most high energy particles are screened out just as all particles below a few hundred $\mathrm{MeV}$ are screened out from the Earth environment by Solar modulation. The strength and spatial distribution of the $k^{-2}$ spectrum of magnetic irregularities have to allow this condition - otherwise this picture fails completely

And, a fortiori, all galaxies with a star formation rate similar to ours or even more, should have such a wind, building large cavities around them. If true, the sheets of the galaxy distribution should look like slices of Swiss cheese in gas, with the "holes" of order a Mpc across. The next galaxy similar to ours in such a concept is probably M81, about 3 Mpc distant.

Recent simulations about propagation in our Galaxy also exist from [112].

\section{Generalization: Bottom-Up}

Nothing stated above is completely certain, we are at the beginning of a beautiful era of deeper discovery, and so all the alternatives need to be explored.

- Distant radio galaxies with beam dump, such as $3 \mathrm{C} 147$, e.g. [79. The production of neutrinos has generated a flurry of contradictory papers recently, 8698980 .

- Very high energy neutrinos: prediction (work by $\mathrm{T}$. Weiler et al.): possible correlation with distant sources, characteristic behaviour near horizon - could come from mountains near horizon.

- Magnetic monopoles, e.g., [128]?

- Prediction: Correlation beyond GZK sphere

- other particles may be created in p-p collisions (e.g. work by Farrar et al.)

- Pulsars??? Prediction: Characteristic distribution in Galactic plane, little correlation with supergalactic plane

- Many tests with those cosmic rays up to $310^{18} \mathrm{eV}$

\section{Beautiful main alternative: Top-down}

There is a whole world of models that suggests that the high energy particles can be interpreted as decay products from even more energetic or more massive particles, the top-down scenario, most intensely explored recently by E.-J. Ahn, A. Olinto and G. Sigl.

- Topological defects (work by Sigl et al.): prediction: rising spectrum, large gamma ray background, 1920112 .

- If Quantum gravity scale near TeV: then creation of small black holes and branes possible in atmosphere, [127.

- Prediction: Odd behaviour near horizon should come from open space in East (AUGER)

- different from ultra high energy neutrinos, 364

- if dark matter decay, then correlation with DM distribution, e.g., 171815

- Radically New Physics? Lorentz Invariance Violation? 1029611411511697118 


\section{Tasks}

All such predictions lead to specific tasks, which can be undertaken now or in the near future.

- Assume the various source classes, and test - include small number graininess of source distribution

- Quiescent black holes, active black holes

- Test Galactic wind halo magnetic field

- Test supergalactic sheet magnetic field

- Test correlation with DM distribution

- Better understanding of the fluctuations

- Better understanding of fluorescence

- New experiments: AUGER, EUSO, OWL

- New experimental techniques: Radio emission: LOFAR/SkyView, 48 74; also infrared and acoustic detection methods may be possible - after all most of the energy deposition of an air shower almost certainly goes into heat, some into ionization, visible maybe through radio absorption, and then some obviously into sound wave generation

- new physics such as Lorentz Invariance violation.

- and many more...

\section{Conclusions}

- Events need more statistics, at any energy

- Transition to Galactic cosmic rays

- Spectrum, arrival directions, chemical composition

- ?? New particles

- ?? Galactic magnetic field

- ?? Supergalactic magnetic field

- ?? Maximum energy observed
- ?? Detailed spectral features

- ?? Clustering of arrival directions

- ?? Properties of these clusters

Three main conclusions stand out from all the controversy surrounding the current discussion; apart from just understanding the evolution of the discussion, right back to the early years of last century:

\subsection{Experiment vs. experiment}

We absolutely need an experiment, that checks the different techniques against each other; this will now be the AUGER experiment. There are still fundamental questions, which make a direct comparison between experiments rather difficult, such as ground array versus fluorescence detector - AUGER will hopefully solve that. However, we desperately also need calibration from laboratory experiments, e.g. for the fluorescence yield. In order to understand experiments, theoreticians need to develop the Monte-Carlo simulations, e.g. [6], including the large fluctuation statistics, and also the consistency tests.

\subsection{Theory vs. theory}

Before embarking on proposing a specific class of sources, all the microphysics of such sources should be well developed, using the best data from all other wavelengths. Only when the observed spectrum has been completely fitted, including temporal variability, with however simple a physical model, can we proceed to ask what is the production of very high energy particles, that may come all the way through to us. The cosmological evolution of stellar and non-stellar activity, the formation and evolution of cosmic magnetic fields, all need to be included. Especially cosmic magnetic fields need to be included, despite all the uncertainties about their real strength and spatial distribution.

\subsection{Experiment vs. theory}

Selection effects and systematics can hamper any interpretation of data; theoreticians should carefully listen to the experimentalists, but of course with all due skepticism. The best way is 
to always or at least very often include an experimentalist as coauthor, or have an experimentalist friend vet the paper, to make absolutely sure there is mutual respect and common understanding of what an experiment can really do, and what it maybe cannot do yet. The ultimate solution may be to intermingle theory and experiment in the education of our young students, by sending them around, and including them very early on.

\subsection{Listen!}

We should really listen to Nature, what is the message that Nature is giving us? There is a message hidden in the various manifestations of high energy particles, magnetic fields, interstellar and intergalactic medium. Nature speaks with a consistent voice.

What is the message that Nature is giving us?

\section{Acknowledgements}

P.L. Biermann would like to acknowledge the hospitality during his prolonged stays, first at the University of Maryland, offered by his colleague Eun-Suk Seo in the early spring of 2002, and then at the University of Paris VII, in the late spring 2002, offered by his colleagues Norma Sanchez and Hector de Vega. Work with PLB is being supported through the AUGER theory and membership grant 05 CU1ERA/3 through DESY/BMBF (Germany); further support for the work with PLB comes from the DFG, DAAD, Humboldt Foundation (all Germany), grant 2000/066950 from FAPESP (Brasil) through G. MedinaTanco, KOSEF (Korea), and ARC (Australia). Important input to the discussions in this paper has come from E.-J. Ahn, H. Blümer, M. Chirvasa, A.C. Donea, R. Engel, T. Enßlin, H. Falcke, C. Galea, N. Ikhsanov, K.-H. Kampert, H. Kang, M. Kaufman, P.P. Kronberg, N. Langer, H. Lee, E. Loh, S. Markoff, A. Meli, S. Moiseenko, F. Munyaneza, B. Nath, A. Popescu, R.J. Protheroe, G. Pugliese, W. Rhode, G. Romero, E. Roulet, D. Ryu, N. Sanchez, E.-S. Seo, G. Sigl, T. Stanev, S. Ter-Antonyan, M. Teshima, P. Tinyakov, A. Vasile, H. de Vega, Y. Wang, A. Watson, T. Weiler, St. Westerhoff, and Ch. Zier.

\section{REFERENCES}

1. Ahn, E.-J., Cavaglia, M., Essay submitted on Mar 26, 2002 to the Gravity Research Foundation, hep-ph/0205168

2. Ahn, E.-J., Cavaglia, M., Olinto, A.V., publ. manuscript, hep-th/0201042

3. Alvarez-Muiz, J., Halzen, F., Phys. Rev. D 63, ms. 037302 (2001)

4. Alvarez-Muiz, J., Engel, R., \& Stanev, T., Astrophys. J. 572, 185 - 201 (2002)

5. Alvarez-Muiz, J., Halzen, F., Astrophys. J. Letters 576, L33-L36 (2002), astro-ph/0205408

6. Alvarez-Muiz, J., et al., Phys. Rev. D 66, ms. 033011 (2002)

7. Anchordoqui, L.A., Feng, J.L., Goldberg, H., \& Shapere, A.D., Phys. Rev. D 65, ms. 124027 (2002), hep-ph/0112247

8. Arons, J., Astrophys. J. , submitted (2002), astro-ph/0208444

9. Bahcall, J., Waxman, E., Phys. Rev. D 64, ms. 023002 (2001)

10. Bahcall, J.N., \& Waxman, E., hep-ph/0206217

11. Bednarek, W., Protheroe, R. J., Astropart. Phys. 16, 397 - 409 (2002)

12. Bednarek, W., Giller, M., Zielinska, M., Journ. of Physics G 28, 2283 - 2296 (2002), astro-ph/0205324

13. Bell, A.R., 1978 Month. Not. Roy. Astr. Soc. 182, 147 - 156, and 443 - 455.

14. Berezinskii, V.S., et al., "Astrophysics of Cosmic Rays", North-Holland, Amsterdam (especially chapter IV) (1990).

15. Berezinsky, V., Gazizov, A.Z., \& Grigorieva, S.I., astro-ph/0210095 similar to hep-ph/0204357

16. Bertone, G., Isola, C., Lemoine, M., Sigl, G., Phys. Rev. D 66, ms. 103003 (2002), astro-ph/0209192

17. Bertone, G., Sigl, G., Silk, J., Month. Not. Roy. Astr. Soc. (in press, 2002), astro-ph/0203488

18. Bertone, G., Servant, G., Sigl, G., Phys. Rev. D (submitted, 2002), hep-ph/0211342

19. Bhattacharjee, P. \& Sigl, G., Physics Reports, 327, 109 - 247 (2000), astro-ph/9811011 
20. Bhattacharjee, P., Sigl, G. in "Physics and Astrophysics of Ultra-High-Energy Cosmic Rays", Edited by M. Lemoine, G. Sigl, Lecture Notes in Physics, vol. 576, p. 275 (2001)

21. Biermann, L., Zeitschr. für Astrophys. 29, 274 (1951).

22. Biermann, P.L. \& Strittmatter, P.A., Astrophys. J. 322, 643, (1987).

23. Biermann, P.L., Astron. $\mathcal{E}$ Astroph. 271, 649 (1993), astro-ph/9301008

24. Biermann, P.L. \& and Cassinelli, J.P., Astron. \& Astroph. 277, 691 (1993), astro-ph/9305003

25. Biermann, P.L., \& Strom, R.G., Astron. \& Astroph. 275, 659 (1993), astro-ph/9303013

26. Biermann, P.L., at 23rd International Conference on Cosmic Rays, in Proc. Invited, Rapporteur and Highlight papers; Eds. D. A. Leahy et al., World Scientific, Singapore (1994), p. 45.

27. Biermann, P.L., 1997, Journal of Physics G 23, 1.

28. Biermann, P.L., Langer, N., Seo, E.-S., \& Stanev, T., Astron. \& Astroph. 369, 269 277 (2001)

29. Biermann, P.L., in Cosmic winds and the Heliosphere, Eds. J. R. Jokipii et al., Univ. of Arizona press, p. 887 - 957 (1997), astro-ph/9501030

30. Biermann, P. L.. Sigl, G., in "Physics and Astrophysics of Ultra-High-Energy Cosmic Rays", Edited by M. Lemoine, G. Sigl, Lecture Notes in Physics, 576, p. 1 (2001)

31. Bisnovatyi-Kogan, G.S., Sov. Astron. Astron. J., 14, 652 (1971), orig. Astron. Zh 47, 813 (1970).

32. Blanton, M., Blasi, P., \& Olinto, A. V., Astropart. Phys. 15, 275 - 286 (2001)

33. Breitschwerdt, D. McKenzie, J.F., Völk, H.J., Astron. E Astroph. 245, 79 - 98 (1991)

34. Breitschwerdt, D. McKenzie, J.F., Völk, H.J., Astron. ES Astroph. 269, 54 - 66 (1993)

35. Breitschwerdt, D. \& Komossa, S., in Astrophys. 85 Sp. Sc., Proc. "Astrophys. Dynamics", Eds. D. Berry et al., 272, 3 - 13 (2000)

36. Candia, J., Epele, L., \& Roulet, E., Astropart. Phys. 17, 23 (2002)

37. Candia, J., Roulet, E., \& Epele, L., astro-ph/0206336

38. Candia, J., Mollerach, S., \& Roulet, E., astro-ph/0207143

39. Clarke, T., Kronberg, P.P., Böhringer, H., A New Radio - X-Ray Probe of Galaxy Cluster Magnetic Fields, 2000, Astrophys. J. Letters 547, L111 - L114 (2001), astro-ph/0011281

40. Clay, R. \& Dawson, B., 1998, "Cosmic Bullets" (paperback), by Allen \& Unwin (Australien), preceded by a cloth-bound edition.

41. Dermer, C. D., Astrophys. J. 574, $65-87$ (2002)

42. Distefano, C., Guetta, D., Waxman, E., Levinson, A., Astrophys. J. 575, $378-383$ (2002), astro-ph/0202200

43. Drury, L. O'C., Rep. Progr. Phys. 46, 973 1027 (1983)

44. Dubovsky, S.L., P.G. Tinyakov, P.G., Tkachev, I.I., Phys. Rev. Letters 85, 1154 1157 (2000), astro-ph/0001317

45. Epele, L.N., \& Roulet, E., Phys. Rev. Letters 81, 3295 (1998)

46. Epele, L.N., \& Roulet, E., Journal of High Energy Physics 10, 9 (1998)

47. Epele, L.N., Mollerach, S., \& Roulet, E., Journal of High Energy Physics 03017 (1999)

48. Falcke, H., Gorham, P., Astropart. Phys. (in press) (2002), astro-ph/0207226

49. Farrar, G.F., Biermann, P.L., Phys. Rev. Letters 81, 3579 - 3582 (1998), astro-ph/9806242 and Phys. Rev. Letters 83, 2472 (1999), astro-ph/9901315

50. Fermi, E., 1949, Phys. Rev. 2nd ser., 75, no. $8,1169-1174$

51. Fermi, E., 1954, Astrophys.J. 119, 1 - 6.

52. Gaisser, T.K., Cosmic Rays and Particle Physics, Cambridge Univ. Press (1990)

53. Ginzburg, V.L. \& Syrovatskii, S.I., The origin of cosmic rays, Pergamon Press, Oxford (1964), Russian edition (1963).

54. Glushkov, A.V. , JETP Lett. 75, 1 - 4 (2002)

55. Glushkov, A.V., \& Pravdin, M.I., Astronomy Letters 28, 296 - 302 (2002)

56. Glushkov, A.V., Pravdin, M.I., et al., Physics of Atomic Nuclei 65, 1313 - 1318 (2002)

57. Glushkov, A.V., Pravdin, M.I. , Astronomy Letters 27, 493 - 500 (2002)

58. Glushkov, A.V., Izv. Akad. Nauk Ser. Fiz. 66, 
$101(2002)$

59. Glushkov, A.V., JETP Lett. 73, 313 - 316 (2001)

60. Glushkov, A.V., \& Pravdin, M.I. , JETP Lett. 73, 115 - 117 (2001)

61. Glushkov, A.V., Makarov, I.T. , et al., JETP Lett. 71, 97 - 101 (2000)

62. Gorbunov D.S.,Tinyakov. P.G., Tkachev, I.I., Troitsky, S.V., UNIL-IPT-02-1, (2002) astro-ph/0204360

63. Greisen, K., Phys. Rev. Letters 16, 748 (1966).

64. Halzen, F., Hooper, D., Rep. Prog. Phys., 65, 1025 - 1078 (2002)

65. Halzen, Francis, International Journal of Modern Physics A, 17, 3432 - 3445 (2002).

66. Harari, D., Mollerach, S., \& Roulet, E., Journal of High Energy Physics 08022 (1999)

67. Harari, D., Mollerach, S., \& Roulet, E., Journal of High Energy Physics 02, 035 (2000)

68. Harari, D., Mollerach, S., Roulet, E., Journal of High Energy Physics 0010, 047 (2000), astro-ph/0005483

69. Harari, D., Mollerach, S., Roulet, E., \& Sanchez, F., Journal of High Energy Physics 03045 (2002)

70. Harari, D., Mollerach, S., \& Roulet, E., Journal of High Energy Physics 07006 (2002) Journal of High Energy Physics 07 (2002) 006.

71. Hillas, A. M., Annual Rev. of Astron. \& Astrophys. 22, 425 (1984).

72. The High Resolution Fly's Eye Collaboration, Astropart. Phys. (submitted, 2002), astro-ph/0208301

73. Höflich, P. et al., Astrophys. J. 521, 179 (1999)

74. Huege, T., Falcke, H., in Proc. of the 6th European VLBI Network Symposium, Ros E., Porcas R.W., Lobanov, A.P., \& Zensus, J.A. (eds.), MPIfR, Bonn, Germany (2002), p. 25, astro-ph/0207647

75. Hunter, S. D., et al., Astrophys. J. 481, 205 (1997)

76. Isola, C., Sigl, G., Phys. Rev. D 66, ms. 083002 (2002)

77. Isola, C., Lemoine, M., Sigl, G., Phys. Rev. D 65, ms. 023004 (2002)

78. Jokipii, J. R., Astrophys. J. 313, 842 - 846
(1987)

79. Junor, W. et al., Month. Not. Roy. Astr. Soc. 308, 955-960 (1999)

80. Kalashev, O. E., Kuzmin, V. A., Semikoz, D. V., \& Sigl, G., Phys. Rev. D 65, ms. 103003 (2002)

81. Kardashev, N. S., , Astronomicheskii Zhurnal, 41, 807 (1964)

82. Kronberg, P.P., Rep. Prog. Phys., 57, 325 382 (1994).

83. Kronberg, P.P., Lesch, H., Hopp, U., Astrophys. J. 511, 56 - 64 (1999).

84. Kulsrud, R.M., Annual Rev. of Astron. \& Astrophys. 37, 37 (1999).

85. Lagage, P. O., \& Cesarsky, C. J., Astron. \& Astroph. 125, 249 - 257 (1983)

86. Learned, J.G. \& Mannheim, K., Ann. Rev. Nucl. \& Part. Sci. 50, 679 - 749 (2000)

87. Lemoine, M., Sigl, G., "Physics and astrophysics of ultra-high-energy cosmic rays", Lecture notes in physics, Vol. 576, (2001). Edited by M. Lemoine, and G. Sigl.

88. Lovelace, R.V.E., Nature 262, 649 - 652 (1976).

89. Mannheim, K., Protheroe, R. J., Rachen, J. P., Phys. Rev. D 63, ms. 023003 (2001)

90. Markoff, S., Falcke, H., \& Fender, R., Astron. \& Astroph. 372, L25 - L28 (2001)

91. Mezger, P. G., Duschl, W. J., Zylka, R., Astron. 65 Astroph. Rev. 7, 289 - 388 (1996).

92. Melia, F, Falcke, H., Annual Rev. of Astron. E6 Astrophys. 39, 309 - 352 (2001)

93. Mikhailov, A.A., manuscript (2002),

94. Nagano, M., Watson, A.A., Rev. Mod. Phys., 72, 689 - 732 (2000).

95. Nakamura, T. et al., Astrophys. J. 550, 991 (2001)

96. Olinto, A. V. , Nuclear Physics B Proceedings Supplements 97, 66 - 77 (2001)

97. Olinto, A. V., Nuclear Physics B Proceedings Supplements 110, 434 - 442 (2002)

98. Parker, E.N., Astrophys. J. 128, 664 (1958).

99. Peters, B., Nuovo Cimento Suppl., XIV, 436 - 456 (1959)

100.Peters, B., Nuovo Cimento, XXII, 800 - 819 (1961)

101.Piran, T., Physics Reports 314, 575 (1999). 102.Protheroe, R.J., Meyer, H., Phys. Letters B 
493, 1 - 6 (2000), astro-ph/0005349

103.Protheroe, R.J., Donea, A.C., Reimer, A., Astropart. Phys. (in press, 2002), astro-ph/0210249

104Ptuskin, V.S., Völk, H.J., Breitschwerdt, D., Zirakashvili, V.N.: 1997 A\&A 321, 434

105.Ptuskin, V.S. et al., ICRC 26, ms. OG 3.2.32, vol. 4, p. 291 (1999)

106.Pugliese, G., Falcke, H., Biermann, P.L., Astron. \& Astroph. Letters 344, L37 - L40 (1999), astroph/9903036

107.Pugliese, G., Falcke, H., Wang, Y., Biermann, P.L., Astron. ES Astroph. 358, 409 416 (2000), astro-ph/0003025

108Pugliese, G., Falcke, H., Biermann, P. L., Wang, Y., American Astronomical Society Meeting 199, 12.02, (2001)

109Romero, G. E., Kaufman Bernadó, M. M., \& Mirabel, I. F., Astron. \& Astroph. Letters 393, L61 -L64 (2002)

110Ryu, D., Kang, H., Biermann, P.L., Astron. E Astroph. 335, 19 - 25 (1998), astro-ph/9803275

111.Seemann, H. \& Biermann, P.L., Astron. \&6 Astroph. 327, 273 (1997), astro-ph/9706117

112.Sigl, G., in "Observing Ultrahigh Energy Cosmic Rays from Space and Earth", Edited by Humberto Salazar, Luis Villaseor and Arnulfo Zepeda, AIP Conference Proceedings, 566, 266 - 283 (2001)

113.Sigl, G., in "Physics and Astrophysics of Ultra-High-Energy Cosmic Rays", Edited by M. Lemoine, G. Sigl, Lecture Notes in Physics, 576, 196 (2001)

114.Sigl, G., in "Radio Detection of High Energy Particles", First International Workshop RADHEP 2000, Eds. D. Saltzberg \& P. Gorham, AIP Conference Proceedings, 579, 32 (2001)

115.Sigl, G., Proc. 20th Texas Symposium on relativistic astrophysics, AIP conference proc., 586, p. 832 (2001), Eds. J. C. Wheeler \& H. Martel

116Sigl, G., Science 291, 73 - 79 (2001).

117.Sigl, G., Torres, D. F., Anchordoqui, L. A., Romero, G. E., Phys. Rev. D 63 ms. 081302 (2001)

118.Sigl, G., SF2A-2002: Semaine de
l'Astrophysique Francaise, meeting held in Paris, France, June 24-29, 2002, Eds.: F. Combes and D. Barret, EdP-Sciences (Editions de Physique), Conference Series

119.Smialkowski, A., Giller, M., Michalak, W., Journ. of Physics G 28, 1359 (2002), astro-ph/0203337

120.Stanev, T., Biermann, P.L. \& Gaisser, T.K., Astron. \& Astroph. 274, 902 (1993), astro-ph/9303006

121.Stanev, T., Engel, R., Alvarez-Muiz, J., Seckel, D., Nuclear Physics B Proceedings Supplements, 110, 491 - 493 (2002)

122.Takeda, M., et al., Astropart. Phys. (in press) (2002), astro-ph/0209422

123.Tinyakov, P.G., Tkachev, I.I., JETP Lett. 74, 1 - 5 (2001), Pisma Zh.Eksp.Teor.Fiz. 74, 3 7 (2001), astro-ph/0102101

124.Tinyakov, P.G., Tkachev, I.I., JETP Lett. 74, 445 - 448 (2001), Pisma Zh.Eksp.Teor.Fiz. 74, 499 - 503 (2001), astro-ph/0102476

125.Tinyakov, P.G., Tkachev, I.I., CERN-TH2001-320, (2001), astro-ph/0111305

126.Völk, H. J. \& Biermann, P. L., Astrophys. J. Letters 333, L65 - L68 (1988)

127.Waxman, E., Astrophys. J. Letters (submitted, 2002), astro-ph/0210638

128.Wick, St.D., Kephart, T.W., Weiler, T.J., Biermann, P.L., Astropart. Phys. (in press, 2002), astro-ph/0001233

129.Wiebel-Sooth, B., Biermann, P.L., 1999, Landolt-Börnstein, vol. VI/3c, Springer Verlag, p. 37 - 90 .

130.Yoshiguchi, H., Nagataki, S., Tsubaki, S., Sato, K., submitted to Astrophys. J. , astro-ph/0210132

131.Zatsepin, G. T., \& Kuzmin, V. A., Sov. Phys.JETP Lett., 4, 78, (1966), orig. Pis'ma Zh. Eksp. Teor. Fiz. 4, 114 (1966) 